Daniela Angerame Yela ${ }^{1}$

Nathalia MarChianl ${ }^{2}$

Relato de Caso

Palavras-chave

Gravidez ectópica/eiologia

Cesárea/efeitos adversos

Cicatriz/patologia

Metotrexato/uso terapêutico

Embolização da artéria uterina

Curetagem/métodos

Relatos de casos

Keywords

Pregnancy, ectopic/etiology

Cesarean section/adverse effects

Cicatrix/pathology

Methotrexate/therapeutic use

Uterine artery embolization

Curettage/methods

Case reports

\section{Tratamento conservador da gravidez ectópica em cicatriz de cesárea: relato de caso}

\author{
Conservative management of ectopic pregnancy in cesarean scar: case report
}

\section{Resumo}

A implantação da gravidez na cicatriz de cesárea é considerada uma forma rara de gestação ectópica com uma alta taxa de morbidade e mortalidade. Este tipo de gestação ectópica pode causar complicações graves, em função dos riscos de ruptura e hemorragia volumosa, que pode resultar em histerectomia e comprometimento do futuro reprodutivo da mulher. Reportamos um caso de uma gestação ectópica em cicatriz de cesárea em uma mulher de 28 anos que foi tratada com sucesso com a combinação de três métodos: metotrexate, embolização da artéria uterina e curetagem guiada por ultrassom. Dessa forma foi preservada sua fertilidade.

\section{Abstract}

Implantation of a pregnancy within a cesarean delivery scar is considered to be the rarest form of ectopic pregnancy, with a high morbidity and mortality. Pregnancy in a cesarean delivery scar may cause catastrophic complications which may result in hysterectomy and compromise the reproductive future of a woman. We report an ectopic pregnancy in cesarean scar case in a 28-year old pregnant woman that was treated with success with the association between three treatment modalities (methotrexate, uterine artery embolization and curettage) and preserve her fertility.
Correspondêncio

Daniela Angerame Yela Rua Alexander Fleming, 101 Cidade Universitário (EP: 13083-881 Campinas (SP), Brasil

Recebido 04/03/2013

Aceito com modificações

$03 / 05 / 2013$
Trabalho realizado no Departamento de Tocoginecologia da Universidade Estadual de Campinas - UNICAMP - Campinas (SP), Brasil. 'Professora Doutora do Departamento de Tocoginecologia, Universidade Estadual de Campinas - UNICAMP - Campinas (SP), Brasil. 2Residente do Departamento de Tocoginecologia, Universidade Estadual de Campinas - UNICAMP - Campinas (SP), Brasil. 


\section{Introdução}

A implantação de uma gravidez na cicatriz de cesárea é considerada a apresentação mais rara de uma gravidez ectópica e de elevada morbimortalidade ${ }^{1}$. O primeiro caso foi descrito em 1978 por Larsen e Solomon e sua incidência vem aumentando devido ao aumento das taxas de parto cesárea e maior acurácia da ultrassonografia para diagnóstico ${ }^{2}$. A incidência estimada é de 1 para 1.800 em até 1 para 2.216 gravidezes, com uma taxa de $6,1 \%$ de todas as gestações ectópicas em mulheres com antecedente de uma cesárea anterior ${ }^{3,4}$.

A gravidez na cicatriz de cesárea pode causar complicações catastróficas como ruptura uterina que causa uma grave hemorragia com risco de vida e leva a histerectomia que compromete o futuro reprodutivo 5 .

Atualmente, são descritas inúmeras técnicas de tratamento conservador para este tipo de gestação ectópica, embora não haja consenso sobre a melhor forma delas. Dentre estas modalidades de tratamento podemos citar o metotrexato, a embolização das artérias uterinas, a curetagem, a histeroscopia e a laparoscopia, isolados ou associados ${ }^{6}$.

Relatamos um caso que foi tratado conservadoramente com metotrexato, embolização da artéria uterina e curetagem com sucesso.

\section{Relato do caso}

Gestante de 28 anos, secundigesta com uma cesárea anterior com queixa de atraso menstrual de três meses e sangramento vaginal há onze dias. Foi encaminhada ao Centro de Atenção Integral da Mulher (CAISM), UNICAMP no dia 18 de maio de 2011 com ultra-sonografia de dez dias antes que mostrava gestação de 7 semanas e 1 dia, com batimentos cardíacos fetais (BCF) presentes, com implantação do saco gestacional em região istmo-cervical infiltrando miométrio até serosa. Gestante encontrava-se hemodinamicamente estável, com sangramento discreto via vaginal e colo impérvio ao toque vaginal. Exames laboratoriais normais (hemograma, coagulograma, função renal e hepática), com hemoglobina de $12,1 \mathrm{~g} / \mathrm{dL}$ e $\beta$-hCG $89,722 \mathrm{mUI} / \mathrm{mL}$. A ultra-sonografia deste dia evidenciava gestação de 7 semanas e 1 dia (CCN 10,5mm) com volume uterino de $308,5 \mathrm{~cm}^{3}$, com saco gestacional de $24 \times 17 \times 22 \mathrm{~mm}$ com embrião vivo implantado em topografia de histerorrafia estendendo-se até serosa desta região e presença de hematoma em região fúndica do útero de $58 \times 24 \times 37 \mathrm{~mm}(\mathrm{~V}=65 \mathrm{~mL})$ (Figuras 1 e 2).

No dia seguinte, foi submetida à aplicação de $79 \mathrm{mg}$ de metotrexato intraovular. Após os procedimentos BCF não foram evidenciados. Evoluiu com sangramento e diminuição dos níveis de hemoglobina $(10,6$ g/dL) e então foi submetida à aplicação de $91,5 \mathrm{mg}$ de metotrexato intramuscular. Após, a ultrassonografia mostrava embrião (CCN $9 \mathrm{~mm}$ ) com BCF ausente, com saco gestacional de 32x19x32 mm implantado em região de histerorrafia e hematoma de $95 \times 34 \times 80 \mathrm{~mm}(\mathrm{~V}=137 \mathrm{~mL})$.

Durante seu acompanhamento, a paciente mantinha-se estável, sem sangramento via vaginal e com regressão do hematoma e do saco gestacional a ultrassonografia houve redução dos níveis de $\beta$-hCG (43.369 mUI/mL). Recebeu alta após oito dias de internação com orientação de retorno em uma semana para controle.

A paciente retorna com $\beta$-hCG de 17,908 mUI/mL, mas com aumento do saco gestacional $(37 \times 13 \times 25 \mathrm{~mm})$. Como a paciente encontrava-se estável e com desejo de preservar sua fertilidade, optou-se por acompanhamento clínico.

Após uma semana, a paciente retornou com queixa de sangramento via vaginal intenso com duração de três dias. Ao exame, apresentava-se descorada com sinais vitais estáveis e com colo impérvio ao toque. Apresentava níveis de hemoglobina de $8,6 \mathrm{~g} / \mathrm{dL}$ e

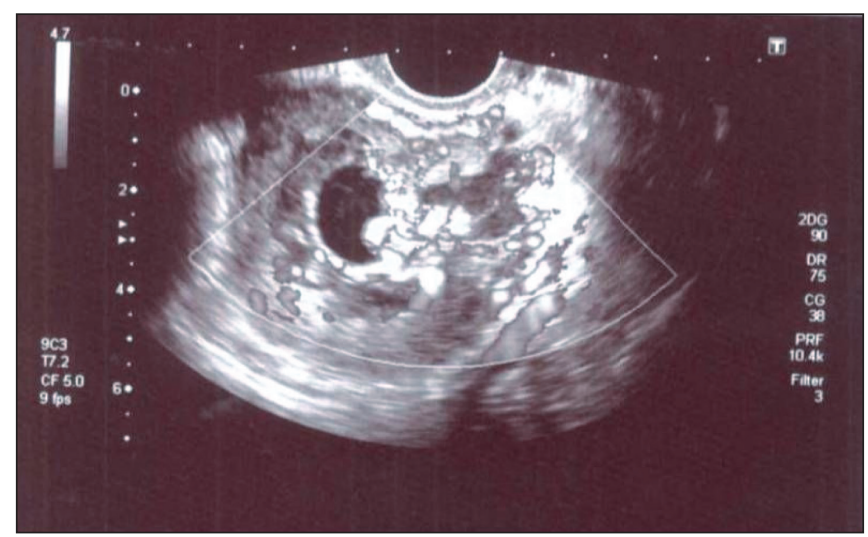

Figura 1. Ultrassonografia mostrando saco gestacional e decídua implantados na topografia de histerorrafia e grande vascularização desta área.

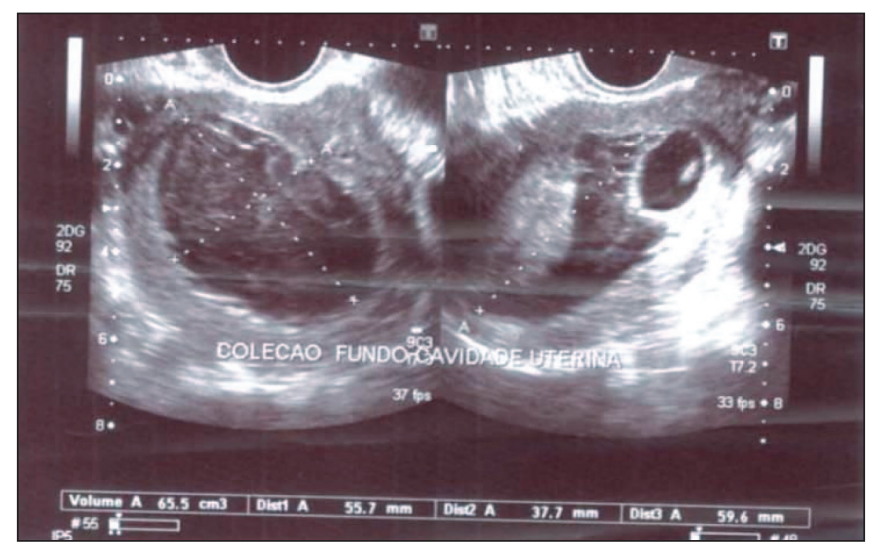

Figura 2. Ultrassonografia mostrando um hematoma em região fúndica do útero. 
imagem ecográfica mantida. A paciente foi internada mantendo sangramento e redução dos níveis de hemoglobina $(7,9 \mathrm{~g} / \mathrm{dL})$ e foi submetida então à curetagem uterina com saída de moderada quantidade de restos e introdução de sonda Foley número 20 com insuflação de $35 \mathrm{~mL}$ de soro no balão no interior do útero para tamponar o sangramento. Devido à hemoglobina de $6,9 \mathrm{~g} / \mathrm{dL}$, a paciente foi submetida à transfusão de três bolsas de sangue. Após o procedimento apresentava hemoglobina de $8,5 \mathrm{~g} / \mathrm{dL}$. Retirada a sonda Foley após 24 horas houve diminuição do sangramento, porém com nova diminuição dos níveis de hemoglobina $(7,6 \mathrm{~g} / \mathrm{dL})$. A ultrassonografia evidenciava um conteúdo complexo com grande vascularização em topografia de istmo de $77 \times 57 \times 77 \mathrm{~mm}$ compatível com coágulos e restos ovulares (Figura 3).

Devido ao desejo da paciente de preservar seu útero, esta foi submetida à embolização das artérias uterinas com boa evolução e diminuição do sangramento e melhora nos níveis de hemoglobina $(8,6 \mathrm{~g} / \mathrm{dL})$. A ultrassonografia após embolização mostrava o mesmo conteúdo em região de istmo com diminuição da vascularização e então a paciente foi submetida à curetagem uterina guiada por ultrassom, sendo necessária transfusão de uma bolsa de sangue durante o procedimento. Recebeu alta no dia seguinte em bom estado geral com discreto sangramento e com hemoglobina de $9 \mathrm{~g} / \mathrm{dL}$ e $\beta$-hCG de $1,026 \mathrm{mUI} / \mathrm{mL}$. Após dois dias da alta, a ultrassonografia mostrava imagem ecogênica em região anterior do útero de $14 \times 17 \times 26 \mathrm{~mm}$, sem fluxo a doplervelocimetria e $\beta$-hCG de $196 \mathrm{mUI} / \mathrm{mL}$ e, após 12 dias, mostrava sinais de processo cicatricial em topografia istmo uterino com $\beta$-hCG de 4,3 mUI/mL. Após oito meses de evolução, a ultrassonografia mostrava-se normal com útero de $68,5 \mathrm{~cm}^{3}$ de volume.

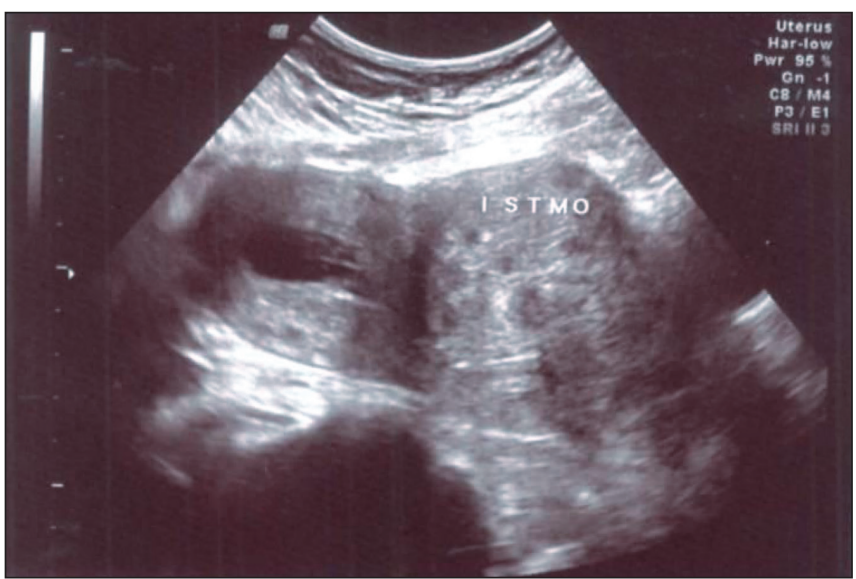

Figura 3. Ultrassonografia mostrando um conteúdo complexo (coágulos e restos uterinos) em istmo uterino.

\section{Discussão}

A gravidez ectópica em cicatriz de cesárea é rara. Desde o primeiro caso descrito em 1978 até 2001, só haviam sido reportados 19 casos, até 2006 havia 155 casos e até 2011 o número de casos descritos na literatura era de 751 , mostrando um rápido aumento na incidência deste tipo de gestação ectópica ${ }^{1,7-10}$.

Desde o advento da ultrassonografia transvaginal, esse tipo de gravidez ectópica, que costumava ser tratada com histerectomia na maioria dos casos por ruptura uterina e intenso sangramento, pôde ser diagnosticada precocemente e tratada de maneira conservadora, preservando a fertilidade $^{11}$. A ultrassonografia apresenta uma sensibilidade de 84,6\% para a detecção deste tipo de gestação ectópica ${ }^{7}$. Mesmo assim, há critérios ultrassonográficos rigorosos para se fazer o diagnóstico diferencial com aborto em curso e gravidez cervical. Dentre estes citamos cavidade uterina vazia, canal cervical vazio, desenvolvimento do saco gestacional na parede anterior do istmo e ausência de tecido miometrial normal entre a bexiga e o saco gestacional ${ }^{11,12}$. Outra forma para o diagnóstico de gestação ectópica em cicatriz de cesárea é a ressonância nuclear magnética ${ }^{13}$.

Várias opções de tratamento conservador têm sido descritas na literatura para preservar a fertilidade das mulheres com esse diagnóstico, embora não haja consenso sobre a melhor modalidade de tratamento. Dentre elas podemos incluir a administração local ou sistêmica de metotrexato, curetagem uterina, histeroscopia, laparoscopia ou laparotomia para retirada do tecido ectópico e embolização de artéria uterina ${ }^{10,14,15}$.

Em uma revisão de literatura, concluiu-se que administração sistêmica de metotrexato é uma alternativa para pacientes estáveis hemodinamicamente e a administração local de metotrexato ou cloreto de potássio guiado por ultrassonografia é outra opção. Após esses procedimentos, deve-se realizar a aspiração cirúrgica do conteúdo guiado por ultrassonografia ${ }^{16}$. O metotrexato pode causar complicações como: leucopenia, alopecia, estomatite, náuseas e vômitos, disfunção hepática ou renal, infecção genital, além de hemorragia vaginal ${ }^{17}$. Um estudo alemão publicou 55 casos de ectópica em cicatriz de cesárea tratadas entre 1996 a 2007 com metotrexato local e sistêmico, dos quais 34 com sucesso ${ }^{18}$. Um estudo recente com esta modalidade de tratamento mostrou que ele é muito efetivo, mas que níveis de $\beta$-hCG maiores que 20,000 necessitam de mais de uma aplicação de metotrexato para resolução do quadro ${ }^{14}$.

Em nosso caso, após a curetagem a paciente apresentou sangramento vaginal intenso que foi controlado parcialmente com uso de sonda Foley. Na literatura, um estudo com 45 mulheres que foram tratadas com metotrexato sistêmico seguido de curetagem e uso de sonda Foley para 
controle do sangramento, mostrou que em apenas três mulheres o tratamento não teve sucesso ${ }^{19}$.

Outra opção de tratamento conservador é a retirada do conteúdo intrauterino por histeroscopia. Foram descritos poucos casos na literatura em gestações ectópicas iniciais ${ }^{6}$. Recentemente, um estudo utilizou a histeroscopia para tratamento da gestação ectópica da cicatriz em 11 pacientes com sucesso sendo que em uma foi associado com embolização uterina e nas outras 10 , associados ao uso de metotrexato ${ }^{20}$. Outra opção para a remoção da gestação ectópica da cicatriz uterina é através da laparoscopia. Para prevenir a hemorragia, os estudos mostram que antes da laparoscopia é realizada a ligadura da artéria hipogástrica bilateralmente, a embolização das artérias uterinas ou a aplicação de vasopressina intra miometrial ${ }^{21}$.

Atualmente, a embolização da artéria uterina vem sendo a opção para o tratamento conservador da gravidez ectópica em cicatriz de cesárea. Essa forma de intervenção pode ter como complicações: tromboembolismo, febre, dor abdominal, infecção pélvica, fistula vesical, atrofia endometrial e falência prematura ovariana ${ }^{7}$. Esta abordagem apresenta um índice de sucesso elevado quando associada à administração de metotrexato, ou apenas associada à curetagem pós embolização $0^{6,15,22-24}$.

Um artigo de revisão no qual se avaliou 22 artigos publicados até 2012 com 239 casos, mostrou uma taxa de sucesso com tratamentos conservadores de 99,1\%. Como em nosso caso, a curetagem uterina foi realizada após 24-72 horas da embolizaçao ${ }^{25}$. A resolução da gestação ectópica em cicatriz de cesárea com tratamento conservador varia de 20 a 75 dias $^{26}$ e a negativação do valor de $\beta$-hCG demora de 30 a 60 dias $^{9,27}$. Em nosso caso, a resolução do quadro foi em 31 dias e a negativação do $\beta$-hCG ocorre em 43 dias.

Recentemente, um artigo chinês mostrou que os sintomas deste tipo de gestação ectópica são inespecíficos e que o diagnóstico é feito pela ultrassonografia e que a melhor escolha de tratamento é a curetagem uterina após uso de metotrexato ou embolização da artéria uterina ${ }^{28}$.

\section{Referências}

1. Fylstra DL, Pound-Chang T, Miller MG, Cooper A, Miler KM. Ectopic pregnancy within a cesarean delivery scar: a case report. Am J Obstet Gynecol. 2002; 187(2):302-4.

2. Bignardi T, Condous G. Transrectal ultrasound-guided surgical evacuation of cesarean scar ectopic pregnancy. Ultrasound Obstet Gynecol. 2010;35(4):481-5.

3. Jurkovic D, Hillaby K, Woelfer B, Lawrence A, Salim R, Elson CJ. First-trimester diagnosis and management of pregnancies implanted into the lower uterine segment Cesarean section scar. Ultrasound Obstet Gynecol. 2003;21 (3):220-7.

4. Seow KM, Huang LW, Lin YH, Lin MY, Tsai YL, Hwang JL. Caesarean scar pregnancy: issues in management. Ultrasound Obstet Gynecol. 2004;23(3):247-53.

5. Herman A, Weinraub Z, Avrech O, Maymon R, Ron-El R, Bukovsky Y. Follow up and outcome of isthmic pregnancy located in a previous caesarean section scar. Br J Obstet Gynaecol. 1995; 102(10):839-41.

6. Litwicka K, Greco E. Caesarean scar pregnancy: a review of management options. Curr Opin Obstet Gynecol. 2011 ;23(6):415-21.

7. Rotas MA, Haberman S, Levgur M. Cesarean scar ectopic pregnancies: etiology, diagnosis, and management. Obstet Gynecol. 2006;107(6):1373-81.

8. Larsen JV, Solomon MH. Pregnancy in a uterine scar sacculus-an unusual cause of postabortal haemorrhage. A case report. S Afr Med J. 1978;53(4): 142-3.

9. Timor-Tritsch IE, Monteagudo A. Unforeseen consequences of the increasing rate of cesarean deliveries: early placenta accreta and cesarean scar pregnancy A review. Am J Obstet Gynecol. 2012;207(1):14-29.

10. Agarwal N, Shahid A, Odejinmi F. Caesarean scar pregnancy (CSP): a rare case of complete scar dehiscence due to scar ectopic pregnancy and its management. Arch Gynecol Obstet. 2013 Jan 5. [Epub ahead of print].
11. Godin PA, Bassil S, Donnez J. An ectopic pregnancy developing in a previous caesarian section scar. Fertil Steril. 1997;67(2):398-400.

12. Arruda MS, Camargo Junior HS. [Cesarean scar ectopic pregnancy: a case report]. Rev Bras Ginecol Obstet. 2008;30(10):518-23

13. Wang $S$, Dong $Y$, Meng X. Intramural ectopic pregnancy: treatment using uterine artery embolization. J Minim Invasive Gynecol. 2013;20(2):241-3.

14. Seow KM, Wang PH, Huang LW, Hwang JL. Transvaginal sono-guided aspiration of gestational sac concurrent with a local methotrexate injection for the treatment of unruptured cesarean scar pregnancy. Arch Gynecol Obstet. 2013 Feb 27. [Epub ahead of print].

15. Shen L, Tan A, Zhu H, Guo C, Liu D, Huang W. Bilateral uterine artery chemoembolization with methotrexate for cesarean scar pregnancy. Am J Obstet Gynecol. 2012;207(5):386.e 1-6.

16. Maymon R, Halperin R, Mendlovic S, Schneider D, Herman A. Ectopic pregnancies in a caesarean scar: review of the medical approach to an iatrogenic complication. Hum Reprod Update. 2004; 10(6):515-23.

17. Li N, Zhu F, Fu S, Shi X. Transvaginal ultrasound-guided embryo aspiration plus local administration of low-dose methotrexate for caesarean scar pregnancy. Ultrasound Med Biol. 2012;38(2):209-13.

18. de Vaate A, Brolmann HA, van der Slikke JW, Wouters MG, Schats R, Huirne JA. Therapeutic options of caesarean scar pregnancy: case series and literature review. J Clin Ultrasound. 2010;38(2):75-84

19. Jiang T, Liu G, Huang L, Ma H, Zhang S. Methotrexate therapy followed by suction curettage followed by Foley tamponade for caesarean scar pregnancy. Eur J Obstet Gynecol Reprod Biol. $2011 ; 156(2): 209-11$.

20. Le A, Shan L, Xiao T, Zhuo R, Xiong H, Wang Z. Transvaginal surgical treatment of cesarean scar ectopic pregnancy. Arch Gynecol Obstet. 2013;287(4):791-6. 
21. Shao $M$, Hu $M X, H u$ M. Temporary bilateral uterine artery occlusion combined with vasopressin in control of hemorrhage during laparoscopic management of cesarean scar pregnancies. J Minim Invasive Gynecol. 2013;20(2):205-8.

22. Wu X, Zhang X, Zhu J, Di W. Caesarean scar pregnancy: comparative efficacy and safety of treatment by uterine artery chemoembolization and systemic methotrexate injection. Eur J Obstet Gynecol Reprod Biol. 2012;161(1):75-9.

23. Yang $X Y, Y u H$, Li KM, Chu $Y X$, Zheng A. Uterine artery embolisation combined with local methotrexate for treatment of caesarean scar pregnancy. BJOG. 2010;117(8):990-6.

24. Lian F, Wang Y, Chen W, Li J, Zhan Z, Ye Y, et al. Uterine artery embolization combined with local methotrexate and systemic methotrexate for treatment of cesarean scar pregnancy with different ultrasonographic pattern. Cardiovasc Intervent Radiol. 2012;35(2):286-91.
25. Zhang B, Jiang ZB, Huang MS, Guan SH, Zhu KS, Qian JS, et al. Uterine artery embolization combined with methotrexate in the treatment of cesarean scar pregnancy: results of a case series and review of the literature. J Vasc Interv Radiol. 2012;23(12):1582-8.

26. Yin X, Su S, Dong B, Ban Y, Li C, Sun B. Angiographic uterine artery chemoembolization followed by vacuum aspiration: an efficient and safe treatment for managing complicated cesarean scar pregnancy. Arch Gynecol Obstet. 2012;285(5):1313-8.

27. Pang YP, Tan WC, Yong TT, Koh PK, Tan HK, Ho TH. Caesarean section scar pregnancy: a case series at a single tertiary centre. Singapore Med J. 2012;53(10):638-42.

28. Zhang Y, Gu Y, Wang JM, Li Y. Analysis of cases with cesarean scar pregnancy. J Obstet Gynaecol Res. 2013;39(1):195-202. 